\title{
Light Scattering Study of Semiflexible Polymer Solutions II. Application of an Integral Equation Theory
}

\author{
Takahiro Sato, Yuji Jinbo, and Akio Teramoto \\ Department of Macromolecular Science, Osaka University, \\ Toyonaka, Osaka 560, Japan
}

(Received September 29, 1994)

\begin{abstract}
The structure factor $\hat{S}(k)$ and the correlation length $\xi$ for semiflexible polymer solutions were formulated using the generalized Ornstein-Zernike (GOZ) integral equation for the monomer-units pair correlation functions. The derived expression for $\hat{S}(k)$ contained the Fourier transform $C(k)$ of the direct correlation function $C(r)$ involved in the GOZ equation. Taking advantage of the short range nature of $C(r)$, we calculated $C(k)$ from the scaled particle theory, which was previously shown to successfully describe thermodynamic quantities of semiflexible polymer solutions. The present theoretical approach gave $\hat{S}(k)$ and $\xi$ which were favorably compared with the previously obtained experimental results for dilute through semidilute solutions of a semiflexible polymer poly( $n$-hexyl isocyanate) dissolved in a good solvent dichrolomethane.
\end{abstract}

KEY WORDS Light Scattering / Semiflexible Polymer / Semidilute Solution

/ Structure Factor / Correlation Length / Generalized Ornstein-Zernike

Integral Equation /

The spatial distribution of monomer-units (or segments) in polymer solutions is not completely random but has some regularity. Owing to the chain connectivity, this regularity ranges over a long distance in comparison with that in low-molar-mass liquids. Light scattering technique provides us with important information about the regularity in the monomerunit distribution in polymer solutions. For example, the range of regularity usually expressed in terms of the correlation length $\xi$ can be measured by light scattering.

In Part 1 of this series, ${ }^{1}$ we made a light scattering study on semidilute solutions of a semiflexible polymer, $\operatorname{poly}(n$-hexyl isocyanate) (PHIC), dissolved in a good solvent, dichloromethane (DCM), and found that $\xi$ as well as the osmotic compressibility $(\partial c / \partial \Pi)$ obtained for these solutions exhibit polymer concentration dependences different from those of flexible polymer-good solvent systems. We succeeded in explaining the concentration dependence of $(\partial c / \partial \Pi)$ for PHIC solutions using the scaled particle theory for wormlike spherocylinders with a weak attractive interaction. On the other hand, the same theory cannot be used for the explanation of $\xi$, which is not a thermodynamic quantity. In Part 1 , we did not give any theoretical interpretation of this spatial property for the semiflexible polymer solution.

In the present study, we apply the generalized Ornstein-Zernike (GOZ) integral equation for the reference interaction site model (RISM) to calculate the structure factor $\hat{S}(k)$ and $\xi$ of semiflexible polymer solutions. The GOZ integral equation approach or the RISM theory is a standard method to deal with the structure of low-molar-mass liquids. ${ }^{2,3}$ This equation however includes an unknown function, the (average) site-site direct correlation function $C(r)$. There are different procedures to determine this function. In this study, we propose to determine the Fourier transform of $C(r)$ 
using the scaled particle theory for wormlike spherocylinders including a weak attractive interaction, which was used in Part 1 to explain the concentration dependence of $(\partial c / \partial \Pi)$ of PHIC solutions. This procedure enables us to explain both $(\partial c / \partial \Pi)$ and $\xi$ (and also $\hat{S}(k)$ ) on the basis of the same molecular model.

Recently Honnell, Curro, and Schweizer ${ }^{4}$ applied the GOZ integral equation to calculate a short-range radial distribution function of monomer units in semiflexible polymer melts. They determined $C(r)$ by solving numerically nonlinear simultaneous equations constructed with the Percus-Yevick closure relation for hard particles, ${ }^{2,3}$ which is a different procedure from ours proposed in this study. While their procedure is suitable to calculate a short-range distribution function in a melt or a very concentrated solution of polymers without any attractive interaction, our procedure is relevant to the treatment of a long-range regularity in semidilute or moderately concentrated polymer solutions where polymer molecules interact each other with both hard-core repulsion and soft attraction. Furthermore, our procedure does not need an involved numerical analysis in distinction from Honnell et al.'s.

In this paper, we first explain the GOZ equation basic to our theoretical development and derive the expressions for $\hat{S}(k)$ and $\xi$. The difference between the present and other light scattering theories are explained in the subsequent section. Finally the results of $\hat{S}(k)$ and $\xi$ calculated by the present theory are compared with the experimental light scattering data for the PHIC-DCM system reported in Part $1 .^{1}$

\section{LIGHT SCATTERING THEORY}

\section{Generalized Ornstein-Zernike Integral Equa- tion}

Let us consider a solution consisting of $n$ monodisperse polymer molecules with the degree of polymerization $N_{0}$. The distribution of monomer units in this solution is specified by the following distribution functions: (1) $N_{0}\left(N_{0}-1\right) / 2$ kinds of intramolecular distribution functions $\omega\left(r ; i_{1}, j_{1}\right)$ defined as the probability density of finding the monomer units $i_{1}$ and $j_{1}$ on one chain 1 separated by a distance $r$ and (2) $N_{0}\left(N_{0}+2\right) / 8$ kinds of intermolecular radial distribution functions $g\left(r ; i_{1}, i_{2}\right)$ defined as the probability density of finding the monomer units $i_{1}$ and $i_{2}$ on two different chains 1 and 2 separated by a distance $r$. [If $N_{0}$ is odd, the number of the different kinds of $g\left(r ; i_{1}, i_{2}\right)$ is $\left(N_{0}+1\right)\left(N_{0}+3\right) / 8$.] In the following discussion, however, the total correlation function $h\left(r ; i_{1}, i_{2}\right)$ defined by $g\left(r ; i_{1}, i_{2}\right)-1$ will be used instead of $g\left(r ; i_{1}, i_{2}\right)$; $h\left(r ; i_{1}, i_{2}\right)$ approaches zero as $r \rightarrow \infty$.

According to Chandler and Andersen ${ }^{5}$ who dealt with the structure of polyatomic molecular liquids using the reference interaction site model, we utilize the following generalized Ornstein-Zernike (GOZ) integral equation for the above polymer solution:

$$
\begin{aligned}
\mathbf{h}(r)= & \int \mathrm{d} \mathbf{r}^{\prime} \int \mathrm{d} \mathbf{r}^{\prime \prime} \omega\left(\left|\mathbf{r}-\mathbf{r}^{\prime}\right|\right) \mathbf{C}\left(\left|\mathbf{r}^{\prime}-\mathbf{r}^{\prime \prime}\right|\right) \\
& \times\left[\omega\left(r^{\prime \prime}\right)+c^{\prime} \mathbf{h}\left(r^{\prime \prime}\right)\right]
\end{aligned}
$$

where $c^{\prime}$ is the number concentration of the polymer and $\mathbf{h}(r)$ and $\boldsymbol{\omega}(r)$ the $N_{0} \times N_{0}$ matrices whose elements are, respectively, the total correlation function $h\left(r ; i_{1}, i_{2}\right)$ and the intramolecular distribution function $\omega\left(r ; i_{1}, j_{1}\right)$ for each pair of monomer units. The elements $C\left(r ; i_{1}, i_{2}\right)$ of the another $N_{0} \times N_{0}$ matrix $\mathbf{C}(r)$ in eq 1 are referred to as the direct correlation functions for the monomers $i_{1}$ and $i_{2}$ on the different polymers 1 and 2 , and they are assumed to be short-range functions which take non-zero values only within a range of $r$ comparable to the monomer size $a$. The GOZ equation was first applied to polymer systems by Schweizer et al. ${ }^{4,6-8}$

Iterative substitutions of eq 1 into $\mathbf{h}\left(r^{\prime \prime}\right)$ on the right-hand side of eq 1 produce an infinite series 


$$
\begin{aligned}
\mathbf{h}(r)= & \int \mathrm{d} \mathbf{r}^{\prime} \int \mathrm{d} \mathbf{r}^{\prime \prime} \omega\left(\left|\mathbf{r}-\mathbf{r}^{\prime}\right|\right) \mathbf{C}\left(\left|\mathbf{r}^{\prime}-\mathbf{r}^{\prime \prime}\right|\right) \omega\left(r^{\prime \prime}\right) \\
& +c^{\prime} \int \mathrm{d} \mathbf{r}^{\prime} \int \mathrm{d} \mathbf{r}^{\prime \prime} \int \mathrm{d} \mathbf{r}^{\prime \prime \prime} \int \mathrm{d} \mathbf{r}^{\prime \prime \prime \prime} \\
& \times \omega\left(\left|\mathbf{r}-\mathbf{r}^{\prime}\right|\right) \mathbf{C}\left(\left|\mathbf{r}^{\prime}-\mathbf{r}^{\prime \prime}\right|\right) \omega\left(\left|\mathbf{r}^{\prime \prime}-\mathbf{r}^{\prime \prime \prime}\right|\right) \\
& \times C\left(\left|\mathbf{r}^{\prime \prime \prime}-\boldsymbol{r}^{\prime \prime \prime \prime}\right|\right) \omega\left(r^{\prime \prime \prime}\right)+\cdots
\end{aligned}
$$

On the right-hand side of this equation, the first and second terms give the contributions of two-chain and three-chain interactions to the total correlation functions, respectively, and the remaining terms contain more than three chain contributions. The first and sec- ond terms can be expressed by the diagrams depicted in Figure 1a, where the direct interaction between particular two monomers is represented by the dashed segment which corresponds to the direct correlation function $C\left(r ; i_{1}, i_{2}\right)$. Therefore eq 1 takes into account interactions among any number of polymer chains in the solution which affect the total correlation functions $h\left(r ; i_{1}, i_{2}\right)$ in $\mathbf{h}(r)$.

However we should notice that eq 1 (or eq 2 ) does not contain the contributions from the following diagrams: (1) the diagrams with the intramolecular interactions within one chain (cf., Figure 1b) and (2) the diagrams with the simultaneous multiple contacts between a pair



First Term

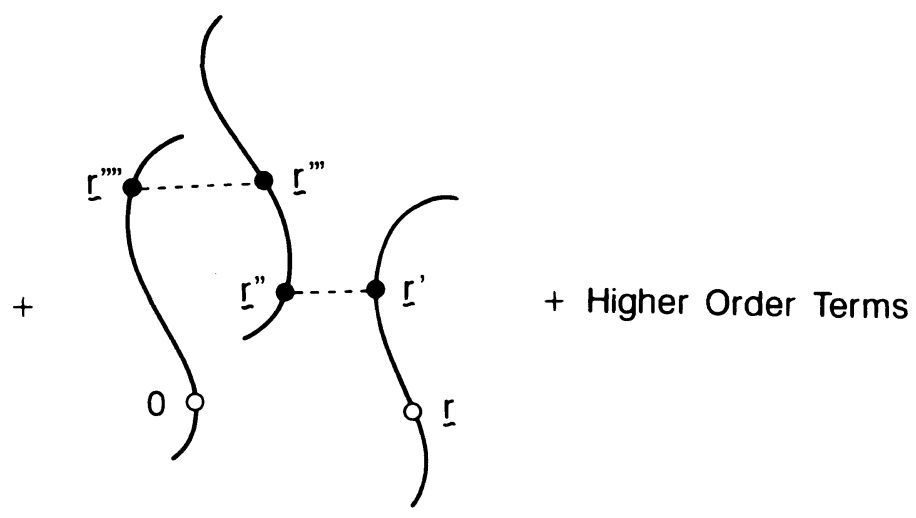

Second Term

(a)
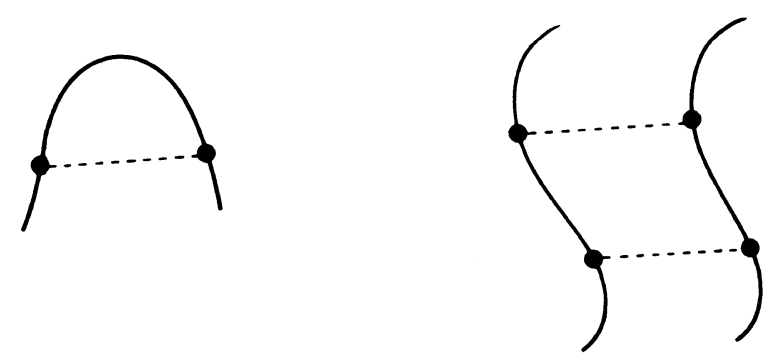

(b)

Figure 1. Diagrammatic expression of the interactions contributing to the structure factor: (a) the interactions considered in the generalized Ornstein-Zernike equation (eq 1); (b) the intramolecular interaction; (c) the simultaneous multiple contacts between pair of chains; the diagrams of (b) and (c) are not included in eq 1 . 
of different chains within the interacting cluster ( $c f$., Figure 1c). Although it is known that, in a good solvent, these diagrams significantly affect $h\left(r ; i_{1}, i_{2}\right)$ and $\omega\left(r ; i_{1}, j_{1}\right)$ for flexible polymers, we can expect that they are much less important for stiff-chain polymers. The monomer density inside one stiff-polymer chain should be low due to its extended conformation, and this low density lowers the probabilities of both intramolecular interaction and the multiple contacts between a pair of different chains. In fact, we know that the intramolecular interaction (or the intramolecular excluded volume effect) little affects the conformation or $\omega\left(r ; i_{1}, j_{1}\right)$ of stiff-chain polymers even in a good solvent, if the chain is sufficiently stiff and the degree of polymerization $N_{0}$ is not very large. ${ }^{1,9}$ In the following discussion, we neglect the contributions of the diagrams shown in Figure $1 \mathrm{~b}$ and $\mathrm{c}$, and utilize the GOZ equation (eq 1) for semidilute solutions of stiff-chain polymers.

\section{Structure Factor}

Equation 1 can be rewritten in the Fourier space using the convolution theorem as

$$
\mathbf{h}(k)=\omega(k) \mathbf{C}(k)\left[\omega(k)+c^{\prime} \mathbf{h}(k)\right]
$$

where $\mathbf{h}(k), \boldsymbol{\omega}(k)$, and $\mathbf{C}(k)$ are the Fourier transforms of the matrices $\mathbf{h}(r), \omega(r)$, and $\mathbf{C}(r)$, respectively. The structure factor $\hat{S}(k)$ is the Fourier transform of the sum of the all (intra- and intermolecular) correlation functions, which can be written in terms of the elements of $\mathbf{h}(k)$ and $\boldsymbol{\omega}(k)$, i.e.,

$$
\begin{array}{r}
\hat{S}(k)=\frac{1}{N_{0}}\left[\sum_{i_{1}, j_{1}=1}^{N_{0}} \omega\left(k ; i_{1}, j_{1}\right)\right. \\
\left.+c^{\prime} \sum_{i_{1}, i_{2}=1}^{N_{0}} h\left(k ; i_{1}, i_{2}\right)\right]
\end{array}
$$

where the argument $k$ has the meaning of the absolute value of the scattering vector. Therefore, in order to formulate $\hat{S}(k)$, we need the expressions of the elements of $\mathbf{h}(k)$ and $\omega(k)$. While the elements of $\boldsymbol{\omega}(k)$ can be calculated from the single chain statistics, $\mathbf{h}(k)$ must be determined from eq 3 , where the $2 \times N_{0}\left(N_{0}+2\right) / 8$ [or $2 \times\left(N_{0}+1\right)\left(N_{0}+3\right) / 8$ for odd $N_{0}$ ] elements of $\mathbf{h}(k)$ and $\mathbf{C}(k)$ are unknown functions. Although Lowden and Chandlar ${ }^{10,11}$ determined $\mathbf{h}(k)$ for small polyatomic molecules (like benzene, carbon tetrachloride, etc.) from eq 3 using Percus-Yevick's closure relation for the direct correlation functions, this procedure is actually intractable for high polymers with large $N_{0}$ due to the mathematical complexity of the matrix integral eq 3 .

However when $N_{0}$ is sufficiently large, the mathematical difficulty may be avoided by using the following approximation. The functions $C\left(k ; i_{1}, i_{2}\right)$ are expected to be essentially independent of $i_{1}$ and $i_{2}$ when the monomers $i_{1}$ and $i_{2}$ are not near the chain ends. Now if we approximate all the elements $C\left(k ; i_{1}, i_{2}\right)$ by the function $C(k)$ averaged over all the monomers, i.e.,

$$
C(k) \equiv N_{0}^{-2} \sum_{i_{1}, i_{2}=1}^{N_{0}} C\left(k ; i_{1}, i_{2}\right)
$$

eq 3 gives

$$
h(k)=\omega(k) C(k)\left[\omega(k)+c_{0}^{\prime} h(k)\right]
$$

where

$$
\begin{gathered}
\omega(k) \equiv N_{0}^{-1} \sum_{i_{1}, j_{1}=1}^{N_{0}} \omega\left(k ; i_{1}, j_{1}\right), \\
h(k) \equiv N_{0}^{-2} \sum_{i_{1}, i_{2}=1}^{N_{0}} h\left(k ; i_{1}, i_{2}\right),
\end{gathered}
$$

and $c_{0}^{\prime}$ the number concentration of the monomer unit in the solution $\left(\equiv N_{0} c^{\prime}\right) ; \omega(k)$ is the single chain structure factor. Equation 6 is identical with the Fourier transform of the original Ornstein-Zernike integral equation for monoatomic molecular liquids. ${ }^{2}$ Curro and Schweizer ${ }^{8}$ showed that the consideration of the end-effect on $C(k)$ in a perturbative way gives a correction term of order of $N_{0}{ }^{-2}$ to $h(k)$, which should be small for polymers with high $N_{0}$. 
Equation 6 is solved for $h(k)$ to give

$$
h(k)=\frac{\omega^{2}(k) C(k)}{1-c_{0}^{\prime} \omega(k) C(k)}
$$

and, from eq 4 , the structure factor $\hat{S}(k)$ is expressed by

$$
\hat{S}(k)=\frac{\omega(k)}{1-c_{0}^{\prime} \omega(k) C(k)}
$$

Therefore we need the two functions $\omega(k)$ and $C(k)$ to calculate $\hat{S}(k)$.

For the reason mentioned in the previous section, the intramolecular interactions depicted in Figure 1b can be neglected for stiff-chain polymers. Therefore the screening effect on the intramolecular excluded volume is not expected to exist for these polymers, and the single-chain structure factor $\omega(k)$ may be assumed to be independent of the polymer concentration. Using the wormlike chain model, several authors calculated $\omega(k)$ at infinite dilution $[\omega(k)$ is equal to the intramolecular interference factor $P(k)$ multiplied by $N_{0}$ ]. In Part $1,{ }^{1}$ we demonstrated that $P(k)$ for PHIC in $\mathrm{DCM}$ at infinite dilution was very nicely fitted by the theoretical results of Yoshizaki and Yamakawa $^{12}$ for the unperturbed wormlike chain model. Therefore we use the same theory for $\omega(k)$ in eq 10 . We must also add that in a large $k$ region Yoshizaki-Yamakawa's theoretical results are more accurate than Koyama's $\operatorname{expression}^{13}$ for $\omega(k)$ which Honnell, Curro, and Schweizer ${ }^{4}$ used in their calculation of $\hat{S}(k)$ for semiflexible polymer melts from the GOZ integral equation.

\section{Expression for $C(k)$}

In order to obtain $C(k)$, we adopt the following procedure. We are interested in the light scattering structure factor in the present study. Therefore our $k$ satisfies the condition $k a \ll 1$ where $a$ is the monomer size. Thus it follows from this condition along with the short-range nature of the direct correlation function that $C(k)$ may be approximated by

$$
C(k) \cong C(0)
$$

in the range of $k$ studied by the light scattering experiment.

The fluctuation theory for the light scattering $^{14}$ gives the relation $\hat{S}(0)=\left(R T / M_{0}\right) /$ $(\partial \Pi / \partial c)$ for binary solutions where $M_{0}$ is the monomer molecular weight, $c$ the polymer mass concentration, and $\Pi$ the osmotic pressure of the solution. Therefore, from eq 10 , the quantity $C(0)$ is related to the thermodynamic quantity $(\partial \Pi / \partial c)$ by the equation

$$
C(0)=\frac{1}{c_{0}^{\prime}}\left[\frac{1}{N_{0}}-\frac{M_{0}}{R T}\left(\frac{\partial \Pi}{\partial c}\right)\right]
$$

In Part $1,{ }^{1}$ we proposed an expression of $(\partial \Pi / \partial c)$ for solutions containing wormlike spherocylinders which interact each other by a hard-core repulsion $u_{0}$ and a weak attractive interaction $w$. Taking the system with $w=0$ as the reference state and $w$ as a thermodynamic perturbation, we obtained

$$
\begin{aligned}
\frac{M_{0}}{R T}\left(\frac{\partial \Pi}{\partial c}\right)= & \frac{M_{0}}{R T}\left(\frac{\partial \Pi}{\partial c}\right)_{w=0} \\
& -\frac{\left\langle\beta_{w}\right\rangle}{N_{0}^{2}} c_{0}^{\prime}+\cdots
\end{aligned}
$$

Here $(\partial \Pi / \partial c)_{w=0}$ is $(\partial \Pi / \partial c)$ for the reference state, i.e., for the wormlike hard spherocylinder system, and $\left\langle\beta_{w}\right\rangle$ the binary cluster integral with respect to the attractive interaction potential $w$. The former quantity can be formulated by the scaled particle theory for wormlike spherocylinders as

$$
\begin{aligned}
& \frac{M_{0}}{R T}\left(\frac{\partial \Pi}{\partial c}\right)_{w=0}=\frac{1}{N_{0}\left(1-v c^{\prime}\right)^{2}} \\
& \quad \times\left[1+\frac{B c^{\prime}}{1-v c^{\prime}}+\frac{2 C c^{\prime 2}}{\left(1-v c^{\prime}\right)^{2}}\right]
\end{aligned}
$$

with 


$$
\begin{aligned}
& B=\frac{\pi}{2} L_{c}^{2} d+6 v \\
& C=\left(v+\frac{\pi}{12} d^{3}\right)\left(\frac{\pi}{2} L_{c}^{2} d+4 v-\frac{\pi}{6} d^{3}\right)
\end{aligned}
$$

where $L_{c}, d$, and $v$ are the cylinder length, diameter, and volume of the spherocylinder hard core, respectively. The quantity $\left\langle\beta_{w}\right\rangle$ is given by

$$
\left\langle\beta_{w}\right\rangle=-\frac{\pi}{2}\left(L_{c}+d\right)^{2} \bar{\delta}
$$

with a parameter $\bar{\delta}$ representing the strength of the attractive interaction ( $c f$. , eq A3 of ref 1). We should notice that eq 14 and 16 do not consider effects coming from the diagrams depicted in Figure $1 \mathrm{~b}$ and $\mathrm{c}$, just like the GOZ equation.

Schweizer and coworkers ${ }^{4,6-8}$ applied the Percus-Yevick closure relation and calculated numerically $C(k)$ for Gaussian and wormlike chains in melts. However the Percus-Yevick closure relation is applicable only to hard particle systems, so that it is not relevant to systems where solutes interact each other not only by the hard-core repulsion but also by some soft attractive interaction like $\operatorname{poly}(n-$ hexyl isocyanate) molecules in DCM. ${ }^{1} \mathrm{Al}$ though Honnell et al. ${ }^{4}$ proposed to use an effective hard-core diameter of the semiflexible polymer to consider the soft-core potential, this treatment may not be necessarily justified. Our above procedure can incorporate the soft potential effect into $C(k)$ more properly. Furthermore while Curro et al.'s procedure needs some troublesome numerical analysis to obtain $C(k)$, our procedure can calculate $C(k)$ from the analytical equations (eq 11-16). It is noted that, for the hard sphere system, the scaled particle theory of Reiss et al. ${ }^{15}$ gives the same equation of state as that from Wertheim's exact solution ${ }^{16}$ for the OrnsteinZernike integral equation combined with the
Percus-Yevick closure relations. This assures that our procedure gives the identical $C(0)$ with Curro et al.'s (at least) in the hard sphere limit.

\section{Correlation Length}

From eq $10-12$, the correlation length $\xi$ for semiflexible polymer solutions can be calculated from the equation ( $c f$., eq 2 of ref 1$)$

$$
\xi^{2}=\frac{1}{3}\left\langle S^{2}\right\rangle \frac{R T}{M}\left(\frac{\partial c}{\partial \Pi}\right)
$$

where $\left\langle S^{2}\right\rangle$ is the mean square radius of gyration at infinite dilution. The osmotic compressibility $(\partial c / \partial \Pi)$ can be calculated from eq $13-16$.

Ohta and Nakanishi ${ }^{17}$ presented a renormalization group theory for semidilute solutions consisting of a fully flexible polymer and a good solvent. Their result can be written in the form*1

$$
\xi^{2}=\frac{1}{3} K(X)\left\langle S^{2}(c)\right\rangle \frac{R T}{M}\left(\frac{\partial c}{\partial \Pi}\right)
$$

where $\left\langle S^{2}(c)\right\rangle$ is the radius of gyration of the chain at the polymer mass concentration $c$ which depends on $c$ due to the screening effect of the intramolecular excluded volume, and the function $K(X)$ expresses the effect on $\xi$ of the multiple contact between a pair of different chains ( $c f$. , Figure 1c); the parameter $X$ is proportional to $c$ divided by the overlap concentration $c^{*}$ or the second virial term. Ohta and Nakanishi showed that $K(X)$ changes from 1 to 0.376 with increasing $X$ or $c$. This indicates an important role of the diagrams like in Figure $1 \mathrm{c}$ in $\xi$ for flexible polymer solutions.

Comparing this Ohta-Nakanishi's expression of $\xi$ for fully flexible polymer solutions with our result (eq 17), we find for semiflexible polymer solutions the relations $K(X)=1$ and $\left\langle S^{2}(c)\right\rangle=\left\langle S^{2}\right\rangle$ irrespective of the polymer concentration. These relations correspond to the neglects of the diagrams shown in Figure $1 \mathrm{~b}$ and c. Equation 17 predicts a weaker con-

*1 Our definition of the correlation length is different from Ohta-Nakanishi's by the factor $3^{1 / 2}$. 
centration dependence of $\xi$ for semiflexible polymers than that for flexible polymers.

\section{COMPARISON WITH OTHER THEORIES}

Recently Shimada, Doi, and Okano ${ }^{18}$ calculated $\hat{S}(k)$ for stiff-chain polymer solutions based on a generalized random phase approximation. In the theory, they utilized a basic integral equation for the response function which is directly related to the segment correlation function and then to $\hat{S}(k)$. Their integral equation has the same form as our GOZ equation (eq 1), if their response function and interaction potential are replaced by $\mathbf{h}(r)+\boldsymbol{\omega}(r)$ and $\mathbf{C}(r)$, respectively. Therefore the mathematical structure of their theory is very similar to ours. (As mentioned in the appendix of ref 18 , their theory considered exactly the same diagrams as in the present study; $c f$., Figure 1.)

However a significant difference residues in the treatment of the nematic (or orientationdependent) interaction between segments or monomers. They specified the segment configuration by the position vector and the orientation vector and considered the orientation dependence of the segment-segment interaction using a phenomenological potential $w\left(\mathbf{r}, \mathbf{u}, \mathbf{u}^{\prime}\right)$ given by

$$
w\left(\mathbf{r}, \mathbf{u}, \mathbf{u}^{\prime}\right)=v_{0} \delta(\mathbf{r})-v_{1}\left[\left(\mathbf{u} \cdot \mathbf{u}^{\prime}\right)^{2}-\frac{1}{3}\right] \delta(\mathbf{r})
$$

where $\mathbf{r}$ is the relative position vector, $\mathbf{u}$ and $\mathbf{u}^{\prime}$ the orientation vectors of the two segments, $v_{0}$ an isotropic excluded volume parameter, and $v_{1}$ a nematic interaction parameter; $\delta(\mathbf{r})$ is the delta function. In case $v_{1}$ is not zero, Shimada et al.'s $\hat{S}(k)$ contains a function $R_{0}(\mathbf{k})$ of the scattering vector $\mathbf{k}$, which does not appear in our $\hat{S}(k)$ given by eq 10 . This function expresses a long-range correlation of the segment distribution through the nematic interaction between contact segments on two stiff polymers, which tends to align the two polymer chains and to make closer non-contact segments belonging to the two chains.

Our theory takes into account the nematic interaction through the binary cluster integral $\left\langle\beta_{\mathrm{w}}\right\rangle$ with respect to the attractive interaction, but $\left\langle\beta_{\mathrm{w}}\right\rangle$ introduces no new $k$-dependent functions [like $R_{0}(\mathbf{k})$ ] in eq 10 for $\hat{S}(k)$. This difference comes from different treatments of the nematic interaction between our and Shimada et al.'s theories.

When $v_{1}$ in eq 19 is equal to zero, Shimada $e t$ al.'s $\hat{S}(k)$ is identical with our eq 10 . However we must equate $C(k)$ in eq 10 to the concentration-independent quantity $v_{0}$ to recover Shimada et al.'s result. This corresponds to the second virial approximation to $(\partial \Pi / \partial c)$ in eq 12. Although Shimada et al. claimed that the third and higher virial coefficients are negligible for rodlike polymer solutions, this claim is correct only when the rodlike polymer is infinitely thin.

In 1984, Benoit and Benmouna ${ }^{19}$ generalized Zimm's classical light scattering theory ${ }^{20}$ with the single contact approximation to obtain $\hat{S}(k)$ which has the same form as ours and Shimada et al.'s with $v_{1}=0$. Benoit and Benmouna approximated the Mayer function for the interaction potential between monomers by the delta function, and expressed the function corresponding to $C(k)$ in eq 10 in terms of some excluded volume parameters.

From the above comparison, it can be said that the form of $\hat{S}(k)$ given by eq 10 is rather general irrespective of the formalism. However the expression of the function corresponding to $C(k)$ in eq 10 depends on the interaction potential form assumed. The approximation of the interaction potential or the Mayer function by the delta function (in Shimada et al.'s and Benoit and Benmouna's theories) loses detail information about the hard-core and attractive interactions between monomers. In this respect, our theory is more detailed than the other two theories. 


\section{COMPARISON WITH EXPERIMENTAL RESULTS}

In this section, we compare our theory (i.e., eq 10 with eq $11-16$ and 17) with the experimental data of the structure factor $\hat{S}(k)$ and the correlation length $\xi$ for dilute through semidilute solutions of a stiff-chain polymer poly(n-hexyl isocyanate) (PHIC) dissolved in a good solvent dichloromethane (DCM) which were reported in Part $1 .{ }^{1}$ In order to calculate $\hat{S}(k)$ and $\xi$ from eq $10-16$ and 17 , we need three quantities $(\partial c / \partial \Pi), \omega(k)$ (or $P(k))$, and $\left\langle S^{2}\right\rangle$; as mentioned above, the latter two quantities can be assumed to be equal to those at infinite dilution. In Part 1, we have already demonstrated that these three quantities are accurately described by theories for the wormlike chain or wormlike spherocylinder model ( $c f$., sections 4.1 and 4.3 of ref 1 ). Therefore if the three quantities are calculated from these theories using the wormlike chain parameters determined previously, ${ }^{1}$ no adjustable parameters remain in eq $10-16$ and 17 .

Figures 2-4 compares the theoretical $\hat{S}(k)$ calculated from eq $10-12$ with the experi-

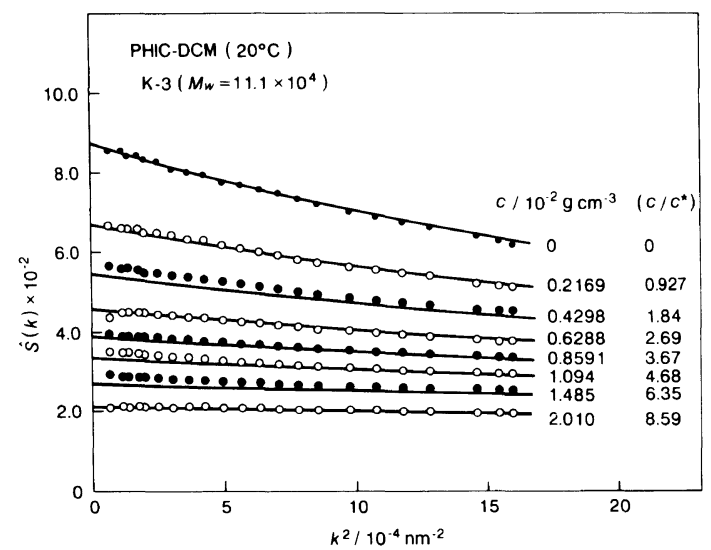

Figure 2. Comparison of experimental structure factor (circles) for DCM solutions of PHIC with $M_{w}=11.1 \times 10^{4}$ with the theoretical one (solid curves) obtained from eq 10-16 using the molecular parameters: the molar mass per contour length $M_{\mathrm{L}}=740 \mathrm{~nm}^{-1}$, persistence length $q=21 \mathrm{~nm}$, hard-core diameter $d=1.07 \mathrm{~nm}$, and strength of the attractive interaction $\delta=-0.36 \mathrm{~nm}$, which were determined in Part $1 .^{1}$

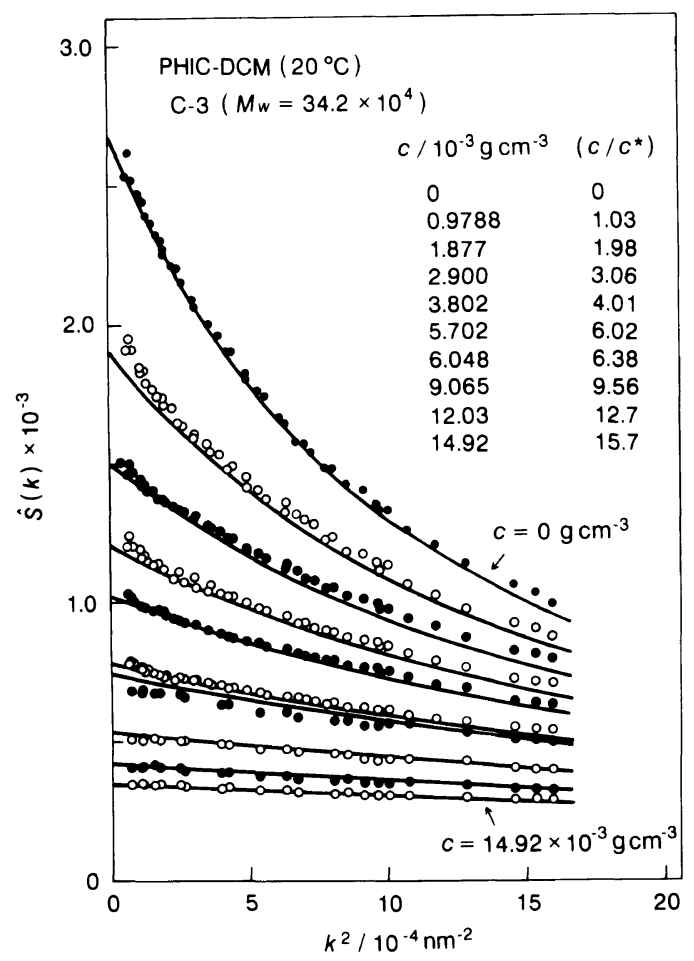

Figure 3. The same comparison as in Figure 2 for PHIC with $M_{w}=34.2 \times 10^{4}$.

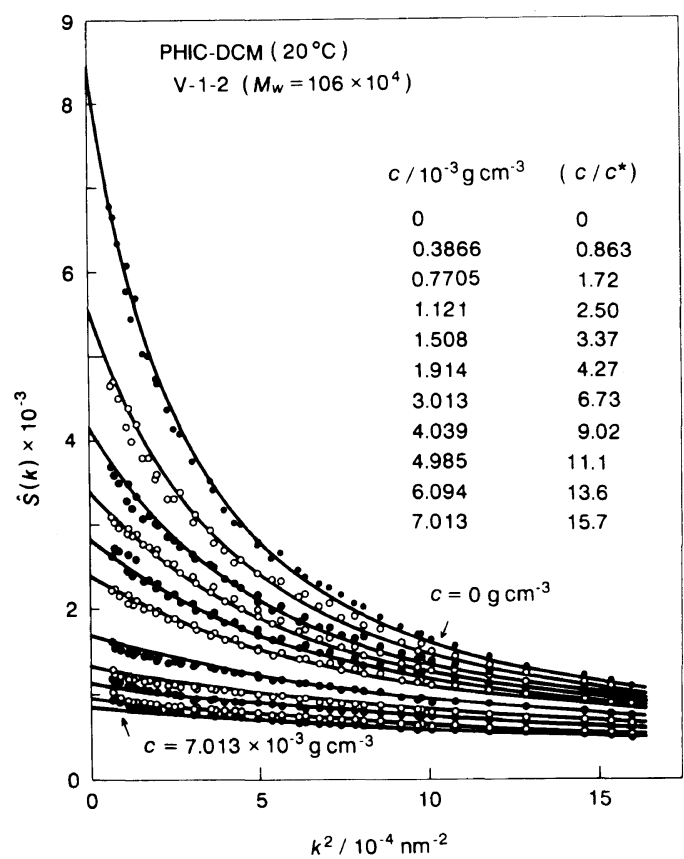

Figure 4. The same comparison as in Figure 2 for PHIC with $M_{w}=106 \times 10^{4}$. 


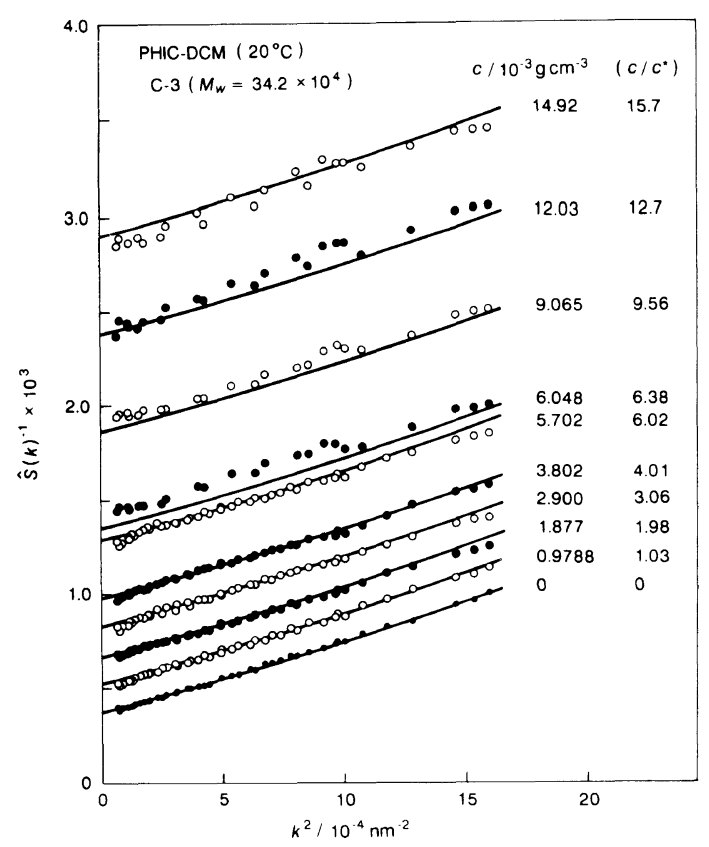

Figure 5. Plot of $\hat{S}(k)^{-1}$ against $k^{2}$; symbols, experimental data shown in Figure 3 for PHIC with $M_{w}=$ $34.2 \times 10^{4}$; solid curves, theoretical values calculated as in Figure 3.

mental $\hat{S}(k)$ for DCM solutions of three PHIC samples with the weight-average molecular weights $M_{w}$ from $11.1 \times 10^{4}$ to $106 \times 10^{4}$. For all the samples displayed, the experimental data represented by the circles are successfully fitted by the theoretical solid curves over the concentration range examined. The agreement between experiment and theory in $\hat{S}(k)$ was also good for four other PHIC samples studied in Part 1, although they are not displayed here.

Since $C(k)$ is taken to be independent of $k$ from eq 11 , the $k$-dependent term of $\hat{S}(k)^{-1}$ given by eq 10 is only $\omega(k)^{-1}$ which is independent of $c$. Therefore the plots of $\hat{S}(k)^{-1}$ $v s . k^{2}$ for different $c$ should be represented by curves with the same shape but with different intercepts. Figure 5 shows the same data for sample C-3 with $M_{w}=34.2 \times 10^{4}$ as in Figure 3 by plotting $\hat{S}(k)^{-1}$ against $k^{2}$. The experimental $\hat{S}(k)$ shown by the circles clearly exhibits this feature.

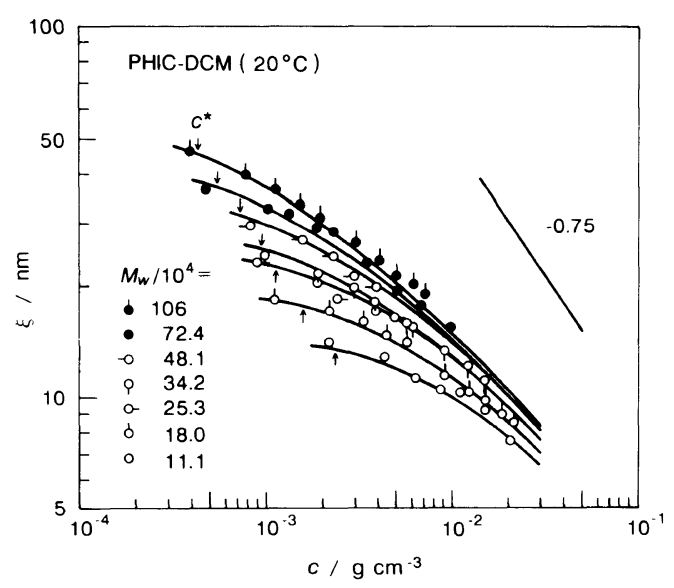

Figure 6. Comparison of the correlation length for the PHIC-DCM system with the present theory (eq 17); arrows, the overlap concentrations for the PHIC samples.

Figure 6 compares the theoretical results of $\xi$ calculated from eq 17 with the experimental $\xi$ for the PHIC-DCM system. Here different circles represent the experimental data for different molecular weight PHIC samples indicated. For each sample, the theoretical solid curve nicely fits the data points. As mentioned in Part $1,{ }^{1}$ it is one of the most significant features that the $c$ dependence of $\xi$ for PHIC solutions is much weaker than the prediction of the scaling $\operatorname{law}^{21}\left(\xi \sim c^{-3 / 4}\right)$ and of the renormalization group theory ${ }^{17}(c f$., Figure 9 of Part 1) for flexible polymer solutions above the overlap concentration $c^{*}$; in Figure 6, the overlap concentrations for the PHIC solutions are indicated by the arrows. This feature comes from weaker $c$ dependences of the three quantities, $K(X),\left\langle S^{2}(c)\right\rangle$, and $(\partial c / \partial \Pi)$ for the stiff-chain polymer-good solvent system than those for the flexible polymer-good solvent system. As mentioned in the previous section, the former two quantities are independent of $c$ in the present theory for stiff-chain polymers, while they are decreasing functions of $c$ in the renormalization group theory ${ }^{17}$ for flexible polymers in good solvents. Furthermore Figure 8 of Part 1 showed that $(\partial c / \partial \Pi)$ for the PHIC-DCM 


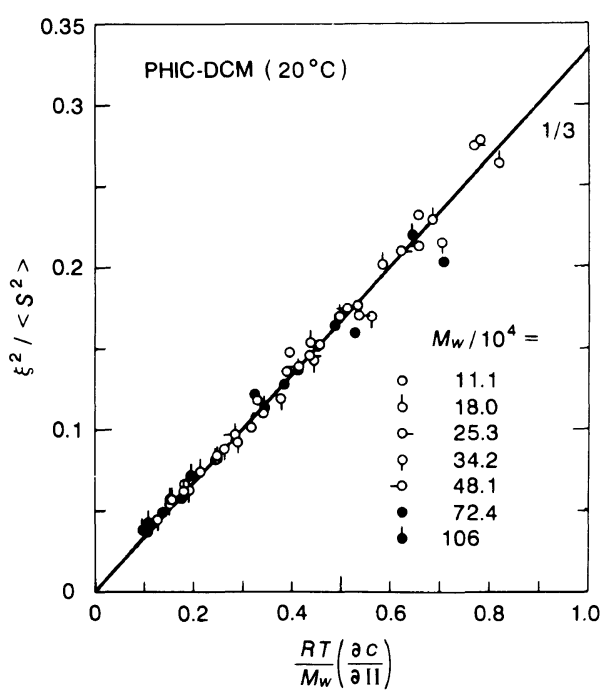

Figure 7. Plot of $\xi^{2} /\left\langle S^{2}\right\rangle$ against $\left(R T / M_{w}\right)(\partial c / \partial \Pi)$ for the PHIC-DCM system; solid line of slope $1 / 3$, theoretical values.

system exhibits a weaker $c$ dependence than that predicted by the renormalization group theory for the flexible polymer ${ }^{22,23}$ in a semidilute region.

Equation 17 can be compared with experimental data in a different way. Figure 7 shows the plot of $\xi^{2} /\left\langle S^{2}\right\rangle$ against $\left(R T / M_{w}\right)(\partial c / \partial \Pi)$ for all the PHIC samples. Here the circles represent the results obtained from experimental data of $M_{w},\left\langle S^{2}\right\rangle, \xi$, and $(\partial \Pi / \partial c)$ presented in Part 1. As predicted by eq 17, the data points for all the samples closely follow the straight line with the slope of $1 / 3$ and the zero intercept. This demonstrates clearly that $\left\langle S^{2}(c)\right\rangle K(X)$ is independent of concentration for the PHIC chain in DCM probably due to the concentration independence of the two quantities.

\section{CONCLUDING REMARKS}

In this study, we have applied the GOZ integral equation to semiflexible polymer-good solvent systems to formulate the structure factor and correlation length for the systems. This theoretical approach is based on the following assumptions:

(1) The polymer chain conformation is not perturbed by the intramolecular excluded volume effect even in a good solvent ( $c f$. Figure 1b).

(2) As a result of the assumption (1), the screening of the intramolecular excluded volume effect by the intermolecular interaction does not take place in semidilute solutions, and then the single-chain conformation is independent of polymer concentration.

(3) The probability of simultaneous multiple contacts between two polymer chains as illustrated in Figure 1c is negligibly small.

The favorable comparison of the present theoretical approach with the spatial properties of PHIC-DCM solutions shown above validates that the above assumptions are fulfilled for this semiflexible-good solvent system. This fulfillment may be due to a very low monomer density inside one PHIC chain given by $3 N_{0} / 4 \pi\left\langle S^{2}\right\rangle$; this low monomer density reduces the probabilities of the intramolecular monomer contact as well as of the multiple contact between two polymer chains. It is well known that the above assumptions are not fulfilled for flexible polymer-good solvent systems. Therefore the present (classical) approach may not be applicable to flexible polymer systems.

If the degree of polymerization of a semiflexible polymer increases very much, intrachain monomer contacts and interchain multiple contacts may take place in the polymer solution. In fact, for PHIC with very high molecular weights, $\left\langle S^{2}\right\rangle$ deviates upward from the unperturbed value $\left(M_{w} \gtrsim 3 \times 10^{6}\right),{ }^{24}$ and also the second virial coefficient exhibits a weak molecular weight dependence $\left(M_{w} \gtrsim 2 \times\right.$ $\left.10^{6}\right)^{24}$ in a good solvent. (In the previous section, we have not used data of $\hat{S}(k)$ and $\xi$ for such high molecular weight PHIC samples.) These are the signs of the breakdown of the above assumptions, so that we may expect some crossover in spatial properties of semiflexible polymer-good solvent systems from 
the stiff-chain behavior to flexible-chain behavior with increasing the molecular weight of PHIC. Such crossover behavior may be an interesting problem to be studied in future.

Acknowledgment. This work was partially supported by a Grant-in-Aid for Scientific Repsearch (No. 06403027) from the Ministry of Education, Science, and Culture of Japan.

\section{REFERENCES}

1. Y. Jinbo, T. Sato, and A. Teramoto, Macromolecules, 27, 6080 (1994).

2. J. P. Hansen and I. R. McDonald, "Theory of Simple Liquids," 2nd ed, Academic Press, London, 1986.

3. C. G. Gray and K. E. Gubbins, "Theory of Molecular Fluids," Vol. 1, Fundamentals, Clarendon Press, Oxford, 1984.

4. K. G. Honnell, J. G. Curro, and K. S. Schweizer, Macromolecules, 23, 3496 (1990).

5. D. Chandler and H. C. Andersen, J. Chem. Phys., 57, 1930 (1972).

6. K. S. Schweizer and J. G. Curro, Phys. Rev. Lett., 58, 246 (1987).

7. J. G. Curro and K. S. Schweizer, Macromolecules, 20, 1928 (1987).
8. J. G. Curro and K. S. Schweizer, J. Chem. Phys., 87, 1842 (1987).

9. T. Norisuye, Prog. Polym. Sci., 18, 543 (1993).

10. L. J. Lowden and D. Chandler, J. Chem. Phys., 59, 6587 (1973).

11. L. J. Lowden and D. Chandler, J. Chem. Phys., 61, 5228 (1974).

12. T. Yoshizaki and H. Yamakawa, Macromolecules, 13, 1518 (1980).

13. R. Koyama, J. Phys. Soc. Jpn., 34, 1029 (1973).

14. H. Yamakawa, "Modern Theory of Polymer Solutions," Harper \& Row, New York, N.Y., 1971.

15. H. Reiss, H. L. Frisch, and J. L. Lebowitz, J. Chem. Phys., 31, 369 (1959).

16. M. S. Wertheim, Phys. Rev. Lett., 10, 321 (1963).

17. T. Ohta and A. Nakanishi, J. Phys. A: Math. Gen., 16, 4155 (1983).

18. T. Shimada, M. Doi, and K. Okano, J. Chem. Phys., 88, 2815 (1988).

19. H. Benoit and M. Benmouna, Polymer, 25, 1059 (1984).

20. B. H. Zimm, J. Chem. Phys., 16, 1093 (1948).

21. M. Daoud, J. P. Cotton, B. Farnoux, G. Jannink, G. Sarma, H. Benoit, R. Duplessix, C. Picot, and P. G. de Gennes, Macromolecules, 8, 805 (1975).

22. T. Ohta and Y. Oono, Phys. Lett., 89A, 460 (1982).

23. P. Wiltzius, H. R. Haller, D. S. Cannell, and D. W. Schaefer, Phys. Rev. Lett., 51, 1183 (1983).

24. H. Murakami, T. Norisuye, and H. Fujita, Macromolecules, 13, 345 (1980). 